TAIWANESE JOURNAL OF MATHEMATICS

Vol. 11, No. 3, pp. 903-914, August 2007

This paper is available online at http://www.math.nthu.edu.tw/tjm/

\title{
WELL-POSEDNESS IN THE GENERALIZED SENSE OF THE FIXED POINT PROBLEMS FOR MULTIVALUED OPERATORS
}

\author{
Adrian Petruşel, Ioan A. Rus and Jen-Chih Yao
}

\begin{abstract}
The purpose of this paper is to define the concept of well-posedness in the generalized sense of a fixed point problem for multivalued operators. Several conditions under which the fixed point problem is well-posed in the generalized sense are given. Some new fixed point theorems are also proved.
\end{abstract}

\section{INTRODUCTION}

Throughout this paper, the standard notations and terminologies in nonlinear analysis are used. For the convenience of the reader we recall some of them here.

Let $(X, d)$ be a metric space. We will use the following symbols:

$$
\begin{aligned}
P(X) & =\{Y \subset X \mid Y \text { is nonempty }\}, P_{b}(X):=\{Y \in P(X) \mid Y \text { is bounded }\} \\
P_{c l}(X): & =\{Y \in P(X) \mid Y \text { is closed }\}, P_{b, c l}(X):=P_{b}(X) \cap P_{c l}(X), \\
P_{c p}(X): & =\{Y \in P(X) \mid Y \text { is compact }\} .
\end{aligned}
$$

If $T: X \rightarrow P(X)$ is a multivalued operator, then

$$
T(Y):=\bigcup_{x \in Y} T(x), \text { for } Y \in P(X)
$$

will denote the image of the set $Y$.

The set of all nonempty invariant subsets of $T$ will be denoted by

$$
I(T):=\{Y \in P(X) \mid T(Y) \subset Y\},
$$

Received March 1, 2007

Communicated by M. H. Shih.

2000 Mathematics Subject Classification: 47H10, 54H25.

Key words and phrases: Pompeiu-Hausdorff functional, The gap functional, Multivalued operator, Fixed point, Strict fixed point, Multivalued generalized contraction, Compact operator. 
while the graph of the multivalued operator $T$ is denoted by

$$
\text { GraphT }:=\{(x, y) \in X \times X \mid y \in T(x)\} .
$$

Also

$$
T^{1}(x):=T(x), \ldots, T^{n+1}(x)=T\left(T^{n}(x)\right), n \in \mathbb{N}, x \in X
$$

denote the iterate operators of $T$.

For $T: X \rightarrow P(X)$ the symbol

$$
F_{T}:=\{x \in X \mid x \in T(x)\}
$$

denotes the fixed point set, while

$$
(S F)_{T}:=\{x \in X \mid\{x\}=T(x)\}
$$

is the strict fixed point set of the multivalued operator $T$.

The following functionals are used in the main section of the paper.

The gap functional

(1) $D_{d}: P(X) \times P(X) \rightarrow \mathbb{R}_{+} \cup\{+\infty\}, D_{d}(A, B):=\inf \{d(a, b) \mid a \in A, b \in B\}$.

The $\delta$ generalized functional

(2) $\delta_{d}: P(X) \times P(X) \rightarrow \mathbb{R}_{+} \cup\{+\infty\}, \delta_{d}(A, B):=\sup \{d(a, b) \mid a \in A, b \in B\}$.

The excess generalized functional

(3) $\rho_{d}: P(X) \times P(X) \rightarrow \mathbb{R}_{+} \cup\{+\infty\}, \rho_{d}(A, B):=\sup \left\{D_{d}(a, B) \mid a \in A\right\}$.

The Pompeiu-Hausdorff generalized functional

(4) $H_{d}: P(X) \times P(X) \rightarrow \mathbb{R}_{+} \cup\{+\infty\}, H_{d}(A, B):=\max \left\{\rho_{d}(A, B), \rho_{d}(B, A)\right\}$.

It is well-known that $\left(P_{b, c l}(X), H_{d}\right)$ is a complete metric space provided $(X, d)$ is a complete metric space.

Also, we denote by

$$
V(Y ; \varepsilon):=\{x \in X \mid D(x, Y)<\varepsilon\}
$$

the $\varepsilon$-neighborhood of the set $Y \in P(X)$.

If $(X, d)$ is a metric space, then $T: X \rightarrow P(X)$ is said to be

(a) closed if $G(T)$ is a closed set in $X \times X$;

(b) compact if $\overline{T(X)}$ is compact.

Also, if $T: X \rightarrow P_{c l}(X)$ is a multivalued operator, then $T$ is called

(i) contractive if $H_{d}(T(x), T(y))<d(x, y)$, for all $x, y \in X$, with $x \neq y$;

(ii) $a$-Lipschitz if $a>0$ and $H_{d}(T(x), T(y)) \leq a d(x, y)$, for all $x, y \in X$; 
(iii) $a$-contraction if it is $a$-Lipschitz with $a \in(0,1)$.

For more details and basic results concerning the above notions see for example $[8,9,13,17]$ and the references therein.

The purpose of this paper is to define the concept of well-posedness in the generalized sense of a fixed point problem for multivalued operators. Several conditions under which the fixed point problem is well-posed are given. Some new fixed point theorems are also proved. The notions and the results of the paper extend and complement some previous ones given in De Blasi, Myjak [2], Lemaire [10], Furi, Vignoli [6], Furi, Martelli, Vignoli [7], Reich, Zaslawski [16], Y.-P. Fang, N.-J. Huang, J.-C. Yao [5], I. A. Rus [19], as well as, from A. Petruşel, I. A. Rus [5] and Yong-hui Zhou, J. Yu, Shu-wen Xiang [24].

\section{Well-Posedness in the Generalized Sense of Fixed Point Problems}

For the beginning let us define the notion of well-posedness in the generalized sense of a fixed point problem.

Definition 2.1. Let $(X, d)$ be a metric space, $Y \in P(X)$ and $T: Y \rightarrow$ $P_{c l}(X)$ be a multivalued operator. Then the fixed point problem is well-posed in the generalized sense (respectively well-posed [15]) for $T$ with respect to $D_{d}$ iff

$\left(a_{1}\right) F_{T} \neq \emptyset$ (respectively $F_{T}=\left\{x^{*}\right\}$ );

$\left(b_{1}\right)$ If $x_{n} \in Y, n \in \mathbb{N}$ and $D_{d}\left(x_{n}, T\left(x_{n}\right)\right) \rightarrow 0$ as $n \rightarrow+\infty$, then there exists a subsequence $\left(x_{n_{i}}\right)$ of $\left(x_{n}\right)$ such that $x_{n_{i}} \stackrel{d}{\rightarrow} x^{*} \in F_{T}$ as $i \rightarrow+\infty$ (respectively $x_{n} \stackrel{d}{\rightarrow} x^{*} \in F_{T}$ as $\left.n \rightarrow+\infty\right)$.

Definition 2.2. Let $(X, d)$ be a metric space, $Y \in P(X)$ and $T: Y \rightarrow$ $P_{c l}(X)$ be a multivalued operator. Then the fixed point problem is well-posed in the generalized sense (respectively well-posed [15]) for $T$ with respect to $H_{d}$ iff

$\left(a_{2}\right)(S F)_{T} \neq \emptyset$ (respectively $\left.(S F)_{T}=\left\{x^{*}\right\}\right)$;

$\left(b_{2}\right)$ If $x_{n} \in Y, n \in \mathbb{N}$ and $H_{d}\left(x_{n}, T\left(x_{n}\right)\right) \rightarrow 0$, as $n \rightarrow+\infty$, then there exists a subsequence $\left(x_{n_{i}}\right)$ of $\left(x_{n}\right)$ such that $x_{n_{i}} \stackrel{d}{\rightarrow} x^{*} \in(S F)_{T}$ as $i \rightarrow+\infty$ (respectively $x_{n} \stackrel{d}{\rightarrow} x^{*} \in(S F)_{T}$ as $n \rightarrow+\infty$ ).

Remark 2.3. It's easy to see that if the fixed point problem is well-posed in the generalized sense for $T$ with respect to $D_{d}$ and $F_{T}=(S F)_{T}$, then the fixed point problem is well-posed in the generalized sense for $T$ with respect to $H_{d}$.

Remark 2.4. If $(X, d)$ is a compact metric space, $Y \in P(X)$ and $T: Y \rightarrow$ $P_{c l}(X)$, then 
(a) the fixed point is well-posed in the generalized sense for $T$ with respect to $D_{d}$ iff

(i) $F_{T} \neq \emptyset$;

(ii) If $x_{n} \in Y, n \in \mathbb{N}$ such that $x_{n} \stackrel{d}{\rightarrow} x^{*}$ and $D_{d}\left(x_{n}, T\left(x_{n}\right)\right) \rightarrow 0$ as $n \rightarrow+\infty$, then $x^{*} \in F_{T}$.

(b) the fixed point is well-posed in the generalized sense for $T$ with respect to $H_{d}$ iff

(i) $(S F)_{T} \neq \emptyset$;

(ii) If $x_{n} \in Y, n \in \mathbb{N}$ such that $x_{n} \stackrel{d}{\rightarrow} x^{*}$ and $H_{d}\left(x_{n}, T\left(x_{n}\right)\right) \rightarrow 0$ as $n \rightarrow+\infty$, then $x^{*} \in(S F)_{T}$.

Clearly, from the well-posedness point of view of a fixed point problem for a multivalued operator $T$, it is of major interest to give sufficient conditions for the continuity of the following functionals: $x \mapsto D_{d}(x, T(x))$ and $x \mapsto H_{d}(x, T(x))$, $x \in Y$. For example, we have the following result.

Lemma 2.5. (E. Llorens Fuster [12]) Let $(X, d)$ be a metric space and $T$ : $X \rightarrow P_{b, c l}(X)$ be a $k$-Lipschitz multivalued operator. Then the functionals $x \mapsto$ $D_{d}(x, T(x))$ and $x \mapsto H_{d}(x, T(x)), x \in X$ are $(k+1)$-Lipschitz. $P_{c l}(X)$.

Remark 2.6. Let $(X, d)$ be a compact metric space, $Y \in P(X)$ and $T: Y \rightarrow$

(a) If $\operatorname{card} F_{T}=1$ and the fixed point is well-posed in the generalized sense for $T$ with respect to $D_{d}$, then the fixed point is well-posed for $T$ with respect to $D_{d}$.

(b) If $\operatorname{card}(S F)_{T}=1$ and the fixed point is well-posed in the generalized sense for $T$ with respect to $H_{d}$, then the fixed point is well-posed for $T$ with respect to $H_{d}$.

For similar definitions see $[15,23,24]$. For the single-valued case, see $[2,10$, $7,11,16,5]$.

Some abstract results are given now.

Lemma 2.7. Let $X$ be a nonempty set and $d, d^{\prime}$ two metrics on $X$. Suppose that $d, d^{\prime}$ are metric equivalent. Let $T: X \rightarrow P_{c l}(X)$ be a multivalued operator. Then

(i) The fixed point problem in the generalized sense for $T$ is well-posed with respect to $D_{d}$ if and only if it is well-posed in the generalized sense for $T$ with respect to $D_{d^{\prime}}$. 
(ii) The fixed point problem in the generalized sense for $T$ is well-posed with respect to $H_{d}$ if and only if it is well-posed in the generalized sense for $T$ with respect to $H_{d^{\prime}}$.

Proof.

(i) Let $c_{1}, c_{2}>0$ such that $d \leq c_{1} d^{\prime}$ and $d^{\prime} \leq c_{2} d$. Then $D_{d} \leq c_{1} D_{d^{\prime}}$ and $D_{d^{\prime}} \leq c_{2} D_{d}$. Let $x_{n} \in X, n \in \mathbb{N}$ be such that $D_{d^{\prime}}\left(x_{n}, T\left(x_{n}\right)\right) \rightarrow 0$, as $n \rightarrow+\infty$. Then:

$$
D_{d}\left(x_{n}, T\left(x_{n}\right)\right) \leq c_{1} D_{d^{\prime}}\left(x_{n}, T\left(x_{n}\right)\right) \rightarrow 0, \text { as } n \rightarrow+\infty .
$$

Since the fixed point problem is well-posed in the generalized sense for $T$ with respect to $D_{d}$, there exists a subsequence $\left(x_{n_{i}}\right)$ of $\left(x_{n}\right)$ such that $x_{n_{i}} \stackrel{d}{\rightarrow} x^{*} \in$ $F_{T}$ as $i \rightarrow+\infty$. As a consequence, we have $d^{\prime}\left(x_{n_{i}}, x^{*}\right) \leq c_{2} d\left(x_{n_{i}}, x^{*}\right) \rightarrow 0$ as $i \rightarrow+\infty$. In a similar way, interchanging the roles of $d$ and $d^{\prime}$, we get the conclusion.

(ii) The second conclusion can be established in a similar way, by taking into account that if $d \leq c_{1} d^{\prime}$ and $d^{\prime} \leq c_{2} d$, then $\delta_{d} \leq c_{1} \delta_{d^{\prime}}$ and $\delta_{d^{\prime}} \leq c_{2} \delta_{d}$.

In a similar way, we have:

Lemma 2.8. Let $X$ be a nonempty set and $d, d^{\prime}$ two metrics on $X$. Suppose that $d, d^{\prime}$ are topologically equivalent (in the sense that they generate the same topology on $X)$ and there exists $c>0$ such that $d \leq c d^{\prime}$. Let $T: X \rightarrow P_{c l}(X)$ be a multivalued operator. Then

(i) if the fixed point problem in the generalized sense for $T$ is well-posed with respect to $D_{d}$ then it is well-posed in large meaning for $T$ with respect to $D_{d^{\prime}}$;

(ii) if the fixed point problem for $T$ is well-posed in the generalized sense for $T$ with respect to $H_{d}$, then it is well-posed in the generalized sense for $T$ with respect to $H_{d^{\prime}}$.

\section{Conditions For Well-Posedness}

In this section, we give several conditions under which the fixed point problem for a multivalued operator is well-posed in the generalized sense.

Our first result, in the setting of a compact metric space, is a very general one.

Theorem 3.1. Let $(X, d)$ be a compact metric space. If $T: X \rightarrow P(X)$ is a closed multivalued operator such that $F_{T} \neq \emptyset$, then the fixed point problem is wellposed in the generalized sense for $T$ with respect to $D$. Moreover, if, additionally, $T$ 
is lower semicontinuous and $(S F)_{T} \neq \emptyset$, then the fixed point problem is well-posed in the generalized sense for $T$ with respect to $H_{d}$.

Proof. Let $x_{n} \in X, n \in \mathbb{N}$ be such that $D_{d}\left(x_{n}, T\left(x_{n}\right)\right) \rightarrow 0$ as $n \rightarrow+\infty$. Let $\left(x_{n_{i}}\right)_{i \in \mathbb{N}}$ be a convergent subsequence of $\left(x_{n}\right)_{n \in \mathbb{N}}$. Suppose $x_{n_{i}} \stackrel{d}{\rightarrow} \widetilde{x}$ as $i \rightarrow+\infty$. Then there exists $y_{n_{i}} \in T\left(x_{n_{i}}\right), i \in \mathbb{N}$, such that $y_{n_{i}} \stackrel{d}{\rightarrow} \widetilde{x}$ as $i \rightarrow+\infty$. Since $T$ is closed, we obtain that $\widetilde{x} \in F_{T}$.

For the second conclusion, let $x_{n} \in X, n \in \mathbb{N}$ be such that $H_{d}\left(x_{n}, T\left(x_{n}\right)\right) \rightarrow 0$ as $n \rightarrow+\infty$. Let $\left(x_{n_{i}}\right)_{i \in \mathbb{N}}$ be a convergent subsequence of $\left(x_{n}\right)_{n \in \mathbb{N}}$. Suppose $x_{n_{i}} \stackrel{d}{\rightarrow} \widetilde{x}$ as $i \rightarrow+\infty$. Since $T$ is continuous, we immediately get that $H_{d}(\widetilde{x}, T(\widetilde{x}))=0$ and hence $\widetilde{x} \in(S F)_{T}$.

Theorem 3.2. If $(X, d)$ is a compact metric space, then for any multivalued contractive operator $T: X \rightarrow P_{c l}(X)$, the fixed point problem is well-posed in the generalized sense with respect to $D_{d}$. Moreover, if additionally $(S F)_{T} \neq \emptyset$, then the fixed point problem is well-posed in the generalized sense with respect to $H_{d}$ too.

Proof. By a theorem of Smithson [22], we have that $F_{T} \neq \emptyset$. Since $T$ is contractive, it is upper semicontinuous and hence closed. The conclusion follows by Theorem 3.1.

Theorem 3.3. Let $(X, d)$ be a metric space and $T: X \rightarrow P(X)$ be a compact contractive multivalued operator.

Then

(i) the fixed point problem for $T$ is well-posed in the generalized sense for $T$ with respect to $D_{d}$;

(ii) if, additionally, $(S F)_{T} \neq \emptyset$, then the fixed point problem for $T$ is well-posed in the generalized sense for $T$ with respect to $H_{d}$.

Proof.

(i) Obviously $F_{T} \neq \emptyset$. Let $\left(x_{n}\right)_{n \in \mathbb{N}}$ be such that $D_{d}\left(x_{n}, T\left(x_{n}\right)\right) \rightarrow 0$ as $n \rightarrow$ $+\infty$. Then there exists $y_{n} \in T\left(x_{n}\right), n \in \mathbb{N}$ such that $d\left(x_{n}, y_{n}\right) \rightarrow 0$ as $n \rightarrow+\infty$. From the compactness hypothesis on $T$ there exists a subsequence $\left(y_{n_{i}}\right)_{i \in \mathbb{N}}$ of $\left(y_{n}\right)_{n \in \mathbb{N}}$ such that $y_{n_{i}} \rightarrow y^{*}$ as $i \rightarrow+\infty$. Hence $x_{n_{i}} \rightarrow y^{*}$ as $i \rightarrow+\infty$. Since $T$ is closed, we obtain that $y^{*} \in F_{T}$.

(ii) Let $\left(x_{n}\right)_{n \in \mathbb{N}}$ be such that $H_{d}\left(x_{n}, T\left(x_{n}\right)\right) \rightarrow 0$ as $n \rightarrow+\infty$. Then there exists $y_{n} \in T\left(x_{n}\right), n \in \mathbb{N}$ such that $d\left(x_{n}, y_{n}\right) \rightarrow 0$ as $n \rightarrow+\infty$. As before, there exists a subsequence $\left(y_{n_{i}}\right)_{i \in \mathbb{N}}$ of $\left(y_{n}\right)_{n \in \mathbb{N}}$ such that $y_{n_{i}} \rightarrow y^{*}$ as $i \rightarrow+\infty$. Hence $x_{n_{i}} \rightarrow y^{*}$ as $i \rightarrow+\infty$. In conclusion $H_{d}\left(y^{*}, T\left(y^{*}\right)\right)=0$ and thus $T\left(y^{*}\right)=\left\{y^{*}\right\}$. 
Let $X$ be a Fréchet space, i. e. a locally convex space which is metrizable and complete. A mapping $\alpha: P_{b}(X) \rightarrow \mathbb{R}_{+}$is called an abstract measure of non-compactness on $X$ if the following conditions hold

(1) (Regularity) $\alpha(A)=0$ implies $\bar{A}$ is compact;

(2) (Convex hull property) $\alpha(\overline{\operatorname{conv}} A)=\alpha(A)$, for each $A \in P_{b}(X)$;

(3) (Non-singularity) $\alpha(A \cup B)=\max \{\alpha(A), \alpha(B)\}$, for each $A, B \in P_{b}(X)$;

(4) (Cantor type property) If $\left(A_{n}\right)_{n \in \mathbb{N}}$ is a decreasing sequence of closed subset of $X$ with $\lim _{n \rightarrow+\infty} \alpha\left(A_{n}\right)=0$, then $\bigcap_{n=1}^{+\infty} A_{n}$ is nonempty and compact.

As consequence, we also have that $\alpha(A) \leq \alpha(B)$ provided $A \subset B$.

Kuratowski $\left(\alpha_{K}\right)$ and Hausdorff $\left(\alpha_{H}\right)$ measures of non-compactness are examples of abstract measures of non-compactness. For other details and related results see Appell [1] and the references therein.

In this setting, a multivalued operator $T: X \rightarrow P(X)$ is said to be densifying with respect to $\alpha$ if $\alpha(T(A))<\alpha(A)$, for each $A \in P_{b}(X) \cap I(T)$ with $\alpha(A)>0$. It is known that compact multivalued operators are densifying with respect to any measure of non-compactness.

We will present now a fixed point result for a densifying multivalued operator. For the single-valued case see Furi and Vignoli [6].

Theorem 3.4. Let $(X, d)$ be a complete metric space and let $T: X \rightarrow P_{b, c l}(X)$ be densifying with respect to $\alpha_{K}$ or $\alpha_{H}$ such that the functional $x \mapsto D_{d}(x, T(x))$ is continuous. Then any bounded sequence $\left(x_{n}\right)_{n \in \mathbb{N}} \in X$ such that $D_{d}\left(x_{n}, T\left(x_{n}\right)\right) \rightarrow$ 0 as $n \rightarrow+\infty$, has a convergent subsequence and all the limit points of $\left(x_{n}\right)_{n \in \mathbb{N}}$ are fixed points of $T$.

Proof. Let $\left(x_{n}\right)_{n \in \mathbb{N}} \in X$ be a bounded sequence such that $D_{d}\left(x_{n}, T\left(x_{n}\right)\right) \rightarrow 0$, as $n \rightarrow+\infty$. Denote $M:=\left\{x_{n}: n \in\{1,2, \cdots\}\right\}$. Then $T(M)=\bigcup_{n \in \mathbb{N}^{*}} T\left(x_{n}\right)$. Since $D_{d}\left(x_{n}, T\left(x_{n}\right)\right) \rightarrow 0$ as $n \rightarrow+\infty$, given any $\varepsilon>0$ the $\varepsilon$-neighborhood $V(T(M) ; \varepsilon)$ of $T(M)$ contains all except a finite number of elements of $M$. Then for each $\varepsilon>0$ we have that

$$
\alpha(M) \leq \alpha(V(T(M) ; \varepsilon)) \leq \alpha(T(M))+2 \varepsilon .
$$

Hence $\alpha(T(M)) \geq \alpha(M)$. This implies that $\alpha(M)=0$ and thus $M$ is compact. Using the continuity of the functional $x \mapsto D_{d}(x, T(x))$, we obtain that all the limit points of $\left(x_{n}\right)_{n \in \mathbb{N}}$ are fixed points of $T$.

As consequence, we can get a well-posedness result. 
Theorem 3.5. Let $(X, d)$ be a bounded and complete metric space and let $T: X \rightarrow P_{b, c l}(X)$ be densifying with respect to $\alpha_{K}$ or $\alpha_{H}$, such that the functional $x \mapsto D_{d}(x, T(x))$ is continuous. Suppose that $\inf _{x \in X} D(x, T(x))=0$. Then the fixed point problem is well-posed in the generalized sense for $T$ with respect to $D_{d}$.

Proof. From the above result we obtain that $F_{T} \neq \emptyset$. Let $\left(x_{n}\right)_{n \in \mathbb{N}} \in X$ be a sequence such that $D_{d}\left(x_{n}, T\left(x_{n}\right)\right) \rightarrow 0$ as $n \rightarrow+\infty$. As in the proof of Theorem 3.4 , we get that $\left(x_{n}\right)_{n \in \mathbb{N}}$ has a subsequence which converges to a fixed point of $T$. The proof is now complete.

Taking into account that any compact multivalued operator is densifying with respect to $\alpha_{K}$ we get the following theorem.

Theorem 3.6. Let $(X, d)$ be a bounded and complete metric space and let $T: X \rightarrow P(X)$ be a compact multivalued operator such that the functional $x \mapsto D_{d}(x, T(x))$ is continuous. Suppose that $\inf _{x \in X} D(x, T(x))=0$. Then the fixed point problem is well-posed in the generalized sense for $T$ with respect to $D_{d}$.

A Krasnoselskii type result can be also established.

Theorem 3.7. Let $(X, d)$ be a complete metric space and let $T_{1}, T_{2}: X \rightarrow$ $P(X)$ be two multivalued operators such that $T_{1}$ is compact and $T_{2}$ is densifying with respect to $\alpha_{K}$ or $\alpha_{H}$. Denote by $T:=T_{1}+T_{2}$ and suppose that $T: X \rightarrow$ $P_{b, c l}(X)$ and that the functional $x \mapsto D_{d}(x, T(x))$ is continuous. Then any bounded sequence $\left(x_{n}\right)_{n \in \mathbb{N}} \in X$, such that $D_{d}\left(x_{n}, T\left(x_{n}\right)\right) \rightarrow 0$ as $n \rightarrow+\infty$, has a convergent subsequence and all the limit points of $\left(x_{n}\right)_{n \in \mathbb{N}}$ are fixed points of $T$.

Proof. From Theorem 3.4 it is sufficient to prove that $T$ is densifying. Let $A \in P_{b}(X)$ such that $\alpha(A)>0$. Then we have

$$
\alpha(T(A)) \leq \alpha\left(T_{1}(A)\right)+\alpha\left(T_{2}(A)\right)=\alpha\left(T_{2}(A)\right)<\alpha(A) .
$$

Of course, a well-posedness result for a Krasnoselskii type multivalued operator can be deduced as before (see Theorem 3.5).

Let us consider now some metrical-type conditions for well-posedness. For some similar results see [15].

Theorem 3.8. Let $(X, d)$ be a complete metric space, $Y \in P_{c l}(X)$ and $T$ : $Y \rightarrow P_{c l}(X)$ be a Ciric-type multivalued operator, $i$. e. there exists $q \in(0,1)$ such that for each $x, y \in Y$

$$
\begin{gathered}
H(T(x), T(y)) \leq q \cdot \max \{d(x, y), D(x, T(x)), D(y, T(y)), \\
\left.\frac{1}{2}(D(x, T(y))+D(y, T(x)))\right\} .
\end{gathered}
$$


If $(S F)_{T} \neq \emptyset$, then the fixed point problem is well-posed for $T$ with respect to $D_{d}$ and with respect to $H_{d}$ too.

Proof. Since $(S F)_{T} \neq \emptyset$ and $T$ is a Ćiric-type multivalued operator, we prove first that $F_{T}=(S F)_{T}=\left\{x^{*}\right\}$. For, let $x^{*} \in(S F)_{T}$. Clearly $(S F)_{T} \subset F_{T}$. Thus, it is enough to prove that $F_{T}=\left\{x^{*}\right\}$. For, let $x \in F_{T}$ with $x^{*} \neq x$. Then

$$
\begin{aligned}
d\left(x^{*}, x\right)=D\left(T\left(x^{*}\right), x\right) \leq & H\left(T\left(x^{*}\right), T(x)\right) \\
\leq & q \cdot \max \left\{d\left(x^{*}, x\right), D\left(x^{*}, T\left(x^{*}\right)\right), D(x, T(x)),\right. \\
& \left.\frac{1}{2}\left(D\left(x^{*}, T(x)\right)+D\left(x, T\left(x^{*}\right)\right)\right)\right\} \\
\leq & q \cdot \max \left\{d\left(x^{*}, x\right), \frac{1}{2}\left(d\left(x^{*}, x\right)+d\left(x, x^{*}\right)\right)\right\} \\
= & q \cdot d\left(x^{*}, x\right) .
\end{aligned}
$$

This contradiction proves that $F_{T}=\left\{x^{*}\right\}$ and hence $F_{T}=(S F)_{T}=\left\{x^{*}\right\}$.

For our purpose let $x_{n} \in Y, n \in \mathbb{N}$ be such that $D\left(x_{n}, T\left(x_{n}\right)\right) \rightarrow 0$ as $n \rightarrow+\infty$. Then

$$
\begin{aligned}
d\left(x_{n}, x^{*}\right) \leq & D\left(x_{n}, T\left(x_{n}\right)\right)+H\left(T\left(x_{n}\right), T\left(x^{*}\right)\right) \leq D\left(x_{n}, T\left(x_{n}\right)\right) \\
& +q \cdot \max \left\{d\left(x_{n}, x^{*}\right), D\left(x_{n}, T\left(x_{n}\right)\right), D\left(x^{*}, T\left(x^{*}\right)\right)\right. \\
& \left.\frac{1}{2}\left(D\left(x_{n}, T\left(x^{*}\right)\right)+D\left(x^{*}, T\left(x_{n}\right)\right)\right)\right\} \\
= & D\left(x_{n}, T\left(x_{n}\right)\right)+q \cdot \max \left\{d\left(x_{n}, x^{*}\right), D\left(x_{n}, T\left(x_{n}\right)\right),\right. \\
& \left.\frac{1}{2}\left(d\left(x_{n}, x^{*}\right)+D\left(x^{*}, T\left(x_{n}\right)\right)\right)\right\} \\
= & D\left(x_{n}, T\left(x_{n}\right)\right)+q \cdot \max \left\{d\left(x_{n}, x^{*}\right), D\left(x_{n}, T\left(x_{n}\right)\right)\right. \\
& \left.d\left(x_{n}, x^{*}\right)+\frac{1}{2} D\left(x_{n}, T\left(x_{n}\right)\right)\right\} \\
= & D\left(x_{n}, T\left(x_{n}\right)\right)+q \cdot \max \left\{D\left(x_{n}, T\left(x_{n}\right)\right), d\left(x_{n}, x^{*}\right)\right. \\
& \left.+\frac{1}{2} D\left(x_{n}, T\left(x_{n}\right)\right)\right\} .
\end{aligned}
$$

Hence

$$
d\left(x_{n}, x^{*}\right) \leq \max \left\{\frac{q+2}{2(1-q)}, 1+q\right\} \cdot D\left(x_{n}, T\left(x_{n}\right)\right) .
$$

Then we immediately get $d\left(x_{n}, x^{*}\right) \leq \frac{q+2}{2(1-q)} \cdot D\left(x_{n}, T\left(x_{n}\right)\right) \rightarrow 0$ as $n \rightarrow+\infty$.

It is an open question if the above result or part of it holds without the assumption $(S F)_{T} \neq \emptyset$. Also, it is of interest to establish a result concerning the 
well-posedness in the generalized sense of the fixed point problem for Ćiric-type multivalued operators.

A consequence of the above theorem is the following well-posedness result for Ćiric-type multivalued operators in Banach spaces.

Corollary 3.9. Let $E$ be a Banach space and $C$ a weakly compact convex subset of it. Let $T: C \rightarrow P_{b, c l}(X)$ be a Ciric-type multivalued operator such that the following assumptions hold:

(i) the functional $x \mapsto H(x, T(x))$ is lower semicontinuous on $C$;

(ii) $\inf _{x \in C} H(x, T(x))=0$.

Then the fixed point problem is well-posed for $T$ with respect to $D$ and $H$.

Proof. From [12] Proposition 3, since $T$ is a Ciric-type multivalued operator, we have that $T$ is strongly $r$-almost convex. From Theorem 2 in [12] we obtain that $(S F)_{T} \neq \emptyset$. The conclusion follows now Therem 3.8.

Another example comes via Kannan nonexpansive multivalued operators. Recall that, if $(X, d)$ is a metric space, then $T: X \rightarrow P_{c l}(X)$ is called a Kannan nonexpansive multivalued operator if for each $x, y \in X$ we have

$$
H_{d}(T(x), T(y)) \leq \frac{1}{2} \cdot\left[D_{d}(x, T(x))+D_{d}(y, T(y))\right] .
$$

It is obvious that a Kannan nonexpansive multivalued operator is not necessary closed. Nevertheless we have:

Theorem 3.10. Let $(X, d)$ be a complete metric space. If $T: X \rightarrow P_{c p}(X)$ is a Kannan nonexpansive multivalued operator such that $\inf _{x \in X} D_{d}(x, T(x))=0$, then the fixed point problem is well-posed in the generalized sense for $T$ with respect to $D_{d}$.

Proof. From Theorem 1 in Shiau, Tan, Wong [21] we have that $F_{T} \neq \emptyset$. Let $x_{n} \in X, n \in \mathbb{N}$ be a sequence such that $D_{d}\left(x_{n}, T\left(x_{n}\right)\right) \rightarrow 0$ as $n \rightarrow+\infty$. Since $T$ is a Kannan nonexpansive multivalued operator, the sequence $\left(T\left(x_{n}\right)\right)_{n \in \mathbb{N}}$ is Cauchy in $\left(P_{c p}(X), H_{d}\right)$. Hence there exists $U^{*} \in P_{c p}(X)$ such that $H_{d}\left(T\left(x_{n}\right), U^{*}\right) \rightarrow 0$ as $n \rightarrow+\infty$. Since $T\left(x_{n}\right), U^{*} \in P_{c p}(X)$ there exist $y_{n} \in T\left(x_{n}\right)$ and $u_{n} \in U^{*}, n \in \mathbb{N}$ such that $d\left(x_{n}, y_{n}\right)=D_{d}\left(x_{n}, T\left(x_{n}\right)\right)$ and $d\left(y_{n}, u_{n}\right)=D_{d}\left(y_{n}, U^{*}\right)$. Moreover, since $U$ is compact, there exists a subsequence $u_{n_{i}}$ of $u_{n}$ that converges to some $u^{*} \in U^{*}$ as $i \rightarrow+\infty$. From $d\left(y_{n}, u_{n}\right) \leq H_{d}\left(T\left(x_{n}\right), U^{*}\right) \rightarrow 0$ as $n \rightarrow+\infty$, we get that $y_{n_{i}} \rightarrow u^{*}$ as $i \rightarrow+\infty$. Since

$$
D_{d}\left(u^{*}, T\left(u^{*}\right)\right)=\lim _{n \rightarrow+\infty} D_{d}\left(y_{n_{i}}, T\left(u^{*}\right)\right) \leq \liminf _{i \rightarrow+\infty} D_{d}\left(T\left(x_{n_{i}}\right), T\left(u^{*}\right)\right)
$$




$$
\leq \liminf _{i \rightarrow+\infty}\left(\frac{1}{2} D_{d}\left(x_{n_{i}}, T\left(x_{n_{i}}\right)\right)\right)+\frac{1}{2} D_{d}\left(u^{*}, T\left(u^{*}\right)\right)=\frac{1}{2} D_{d}\left(u^{*}, T\left(u^{*}\right)\right),
$$

we get that $u^{*} \in F_{T}$. Since $d\left(x_{n}, y_{n}\right)=D_{d}\left(x_{n}, T\left(x_{n}\right)\right) \rightarrow 0$ as $n \rightarrow+\infty$ and $y_{n_{i}} \rightarrow u^{*}$ as $i \rightarrow+\infty$ we immediately obtain that $x_{n_{i}} \rightarrow u^{*}$ as $i \rightarrow+\infty$.

Remark 3.11. From the above proof, it follows that $T\left(x_{n}\right) \stackrel{H_{d}}{\rightarrow} T\left(u^{*}\right)$ as $n \rightarrow+\infty$.

Indeed, $H_{d}\left(T\left(x_{n_{i}}\right), T\left(u^{*}\right)\right) \leq \frac{1}{2} D_{d}\left(x_{n_{i}}, T\left(x_{n_{i}}\right)\right) \rightarrow 0$ as $i \rightarrow+\infty$. Thus $T\left(x_{n_{i}}\right) \stackrel{H_{d}}{\longrightarrow} T\left(u^{*}\right)$ as $n \rightarrow+\infty$ and hence $U^{*}=T\left(u^{*}\right)$.

\section{REFERENCES}

1. J. Appell, Measures of noncompactness, condensing operators and fixed points: an application-oriented survey, Fixed Point Theory 6 (2005), 157-229.

2. F.S. De Blasi, J. Myjak, Sur la porosité des contractions sans point fixe, C. R. Acad. Sci. Paris 308 (1989), 51-54.

3. L. Cirić, Fixed points for generalized multi-valued contractions, Mat. Vesnik 9 (1972), 265-272.

4. H. Covitz, S.B. Nadler jr., Multivalued contraction mappings in generalized metric spaces, Israel J. Math. 8(1970), 5-11.

5. Ya-Ping Fang, Nan-Jing Huang, Jen-Chih Yao, Well-posedness of mixed variational inequalities, inclusion problems and fixed point problems, submitted.

6. M. Furi, A. Vignoli, Fixed points for densifying mappings, Atti Accad. Naz. Lincei Rend. Cl. Sci. Fis. Mat. Natur. 47 (1970) 465-467.

7. M. Furi, M. Martelli, A. Vignoli, On minimum problems for families of functionals, Ann. Mat. Pura Appl. 86 (1970), 181-187.

8. S. Hu and N. S. Papageorgiou, Handbook of Multivalued Analysis, Vol. I-II, Kluwer Academic Publishers, Dordrecht, 1997 and 1999.

9. W. A. Kirk, B. Sims (eds.), Handbook of Metric Fixed Point Theory, Kluwer Acad. Publ., Dordrecht, 2001.

10. B. Lemaire, Well-posedness, conditioning and regularization of minimization, inclusion and fixed-point problems, Pliska Stud. Math. Bulgar. 12 (1998), 71-84.

11. B. Lemaire, C. Ould Ahmed Salem, J. P. Revalski, Well-posedness by perturbations of variational problems, J. Optim. Theory Appl. 115 (2002), 345-368.

12. E. Llorens-Fuster, Set-valued $\alpha$-almost convex mappings, J. Math. Anal. Appl. 233 (1999), 698-712.

13. A. Petruşel, Multivalued weakly Picard operators and applications, Scienticae Mathematicae Japonicae 59 (2004), 167-202. 
14. A. Petruşel, I. A. Rus, Multivalued Picard and weakly Picard operators, Fixed Point Theory and Applications (J. Garcia Falset, E. LLorens Fuster, B. Sims eds.), Yokohama Publishers 2004, pp. 207-226.

15. A. Petruşel, I. A. Rus, Well-posedness of the fixed point problem for multivalued operators, Applied Analysis and Differential Equations (O. Carja, I. I. Vrabie eds.), World Scientific 2007, pp. 295-306.

16. S. Reich and A. J. Zaslavski, Well-posedness of fixed point problems, Far East J. Math. Sci., Special Volume 3 (2001), 393-401.

17. I. A. Rus, Generalized Contractions and Applications, Cluj University Press, 2001.

18. I. A. Rus, Strict fixed point theory, Fixed Point Theory 4 (2003), 177-183.

19. I. A. Rus, Picard operators and well-posedness of fixed point problems, submitted.

20. I. A. Rus, A. Petruşel, G. Petruşel, Fixed Point Theory 1950-2000 : Romanian Contributions, House of the Book of Science, Cluj-Napoca, 2002.

21. C. Shiau, K.-K. Tan and C. S. Wong, A class of quasi-nonexpansive multi-valued maps, Canad. Math. Bull. 18 (1975), 709-714.

22. R. E. Smithson, Fixed points for contractive multifunctions, Proc. A. M. S. 27 (1971), 192-194.

23. H. Yang, J. Yu, Unified approach to well-posedness with some applications, J. Global Optimization 31 (2005), 371-381.

24. Yong-hui Zhou, J. Yu, Shu-wen Xiang, Hadamard types of well-posedness of non-self set-valued mappings for coincide points, Nonlinear Analysis 63 (2005), 2427-2436.

Adrian Petruşel and Ioan A. Rus

Department of Applied Mathematics,

Babeş-Bolyai University Cluj-Napoca,

Kogalniceanu 1,

400084, Cluj-Napoca,

Romania

E-mail: petrusel@math.ubbcluj.ro

Jen-Chih Yao

Department of Applied Mathematic,s

National Sun Yat-sen University,

Kaohsiung 804,

Taiwan, R.O.C.

E-mail: yaojc@math.nsysu.edu.tw 